\title{
SY3-1 Symposium
}

\section{New evidence on optimal management of hypertension}

\section{Sandosh Padmanabhan}

Institute of Cardiovascular \& Medical Sciences, University of Glasgow, UK

Hypertension is the leading cause of death and disability-adjusted life-years worldwide. Lowering systolic blood pressure by $10 \mathrm{mmHg}$ reduces the relative risk of a major cardiovascular event by $20 \%$, with those at highest risk gaining the most in absolute terms. New guidelines have lowered or are considering lowering the threshold for initiation of pharmacologic therapy and BP goals. The reclassification of hypertension is accompanied by new treatment recommendations, with a target blood pressure of $130 / 80 \mathrm{mmHg}$, and treatment at $<140 / 90 \mathrm{mmHg}$ for those at increased risk. Hypertension has multiple causes and limitations of current treatment guidelines are well recgonised - mainly an all-comers approach to treatment; wide inter-individual response to drug classes despite stratification by age/ethnicity; uncertainty in stratification of ethnic minorities other than those of African ancestry; uncertainty in stratification to combination treatment required in most patients. Recent trials like the PATHWAY programme of trials now offer answers contributing to our better understanding of more optimal treatment strategies for hypertensive patients. PATHWAY 1 provides substantial evidence that initial combination therapy achieves more rapid blood pressure reduction safely and with the vast majority of patients reaching target BP within a few weeks of onset of treatment. PATHWAY 2 clearly establishes the case for recommending spironolactone as the optimal fourth line drug in the treatment algorithm for patients with resistant hypertensive patients. PATHWAY 3 provides an important insight into the different metabolic effects of the diuretics commonly used in hypertension treatment strategies and evidence for the benefits of a thiazide/amiloride combination. 